\title{
Oscillatory Behavior on a Three-Node Neural Network Model with Discrete and Distributed Delays
}

\author{
Chunhua Feng \\ College of Mathematics and Statistics, Guangxi Normal University, Guilin 541004, China \\ Correspondence should be addressed to Chunhua Feng; chfeng@mailbox.gxnu.edu.cn
}

Received 23 October 2013; Accepted 1 January 2014; Published 24 February 2014

Academic Editor: Songcan Chen

Copyright (C) 2014 Chunhua Feng. This is an open access article distributed under the Creative Commons Attribution License, which permits unrestricted use, distribution, and reproduction in any medium, provided the original work is properly cited.

This paper investigates the oscillatory behavior of the solutions for a three-node neural network with discrete and distributed delays. Two theorems are provided to determine the conditions for oscillating solutions of the model. The criteria for selecting the parameters in this network are derived. Some simulation examples are presented to illustrate the effectiveness of the results.

\section{Introduction}

Neural networks are complex and large-scale dynamical systems. Time delay is inevitably encountered in implementation of dynamical neural networks and is frequently a source of oscillation and instability. On the one hand, information transmission from one neuron to another neuron may make the response of networks with discrete delays. On the other hand, neural networks usually have spatial extent due to the presence of a multitude of parallel pathways with a variety of axon sizes and lengths. Thus, the distributed delays in neural system need to be considered. In the past two decades, many researches have studied various neural networks with discrete and distributed delays [1-4]. In [1], Gopalsamy and Leung studied the following neural network model:

$$
\begin{aligned}
\frac{d x_{1}(t)}{d t} & =-x_{1}(t)+a \tanh \left[x_{2}(t)-b x_{2}(t-\tau)-c\right], \\
\frac{d x_{2}(t)}{d t}= & -x_{2}(t) \\
& +a \tanh \left[x_{1}(t)-b \int_{0}^{\infty} F(s) x_{1}(t-s) d s-c\right] .
\end{aligned}
$$

By means of the Lyapunov functional, the authors obtained some necessary and sufficient conditions for the existence of a globally asymptotically stable equilibrium point of system (1). Ruan and Filfil [2] considered a two-neuron network model with multiple discrete and distributed delays as follows:

$$
\begin{aligned}
& \frac{1}{a_{10}} \frac{d x_{1}(t)}{d t}+x_{1}(t) \\
& =F\left\{f_{1}+c_{12} x_{2}\left(t-\sigma_{12}\right)\right. \\
& \left.\quad+b_{11} \int_{-\infty}^{t} x_{1}(\tau) k_{11}(t-\tau) d \tau\right\}, \\
& \frac{1}{a_{20}} \frac{d x_{2}(t)}{d t}+x_{2}(t) \\
& =F\left\{f_{2}+c_{21} x_{1}\left(t-\sigma_{21}\right)\right. \\
& \left.\quad+b_{22} \int_{-\infty}^{t} x_{2}(\tau) k_{22}(t-\tau) d \tau\right\},
\end{aligned}
$$

where the delayed feedback kernel satisfies $\int_{0}^{\infty} k_{i i}(s) d s=$ const. and $\int_{0}^{\infty} s k_{i i}(s) d s<\infty, F(u)=\left(1 /\left(1+e^{-u}\right)\right)(i=1,2)$. Local stability analyses are carried out for model (2). Computer simulations are performed to illustrate the obtained 
results. Liao et al. [3] discussed a two-neuron system with distributed delays in the frequency domain as follows:

$$
\begin{aligned}
\frac{d x_{1}(t)}{d t}= & -x_{1}(t) \\
& +a_{1} f\left[x_{2}(t)-b_{2} \int_{0}^{\infty} F(r) x_{2}(t-r) d r-c_{1}\right], \\
\frac{d x_{2}(t)}{d t}= & -x_{2}(t) \\
& +a_{2} f\left[x_{1}(t)-b_{1} \int_{0}^{\infty} F(r) x_{1}(t-r) d r-c_{2}\right],
\end{aligned}
$$

where $F(r)$ is a strong kernel. For simplicity, the authors set $c_{1}=c_{2}=0$. Let

$$
\begin{aligned}
& y_{1}(t)=x_{1}(t)-b_{1} \int_{0}^{\infty} F(r) x_{1}(t-r) d r \\
& y_{2}(t)=x_{2}(t)-b_{2} \int_{0}^{\infty} F(r) x_{2}(t-r) d r .
\end{aligned}
$$

Then system (3) is equivalent to the following model:

$$
\begin{aligned}
\frac{d y_{1}(t)}{d t}= & -y_{1}(t)+a_{1} f\left[y_{2}(t)\right] \\
& -a_{1} b_{2} \int_{0}^{\infty} F(r) f\left[y_{2}(t-r)\right] d r \\
\frac{d y_{2}(t)}{d t}= & -y_{2}(t)+a_{2} f\left[y_{1}(t)\right] \\
& -a_{2} b_{1} \int_{0}^{\infty} F(r) f\left[y_{2}(t-r)\right] d r .
\end{aligned}
$$

By applying the frequency domain approach and analyzing the characteristic equation, the Hopf bifurcation occurs when the mean delay $\mu$ exceeds a critical value. Thus, there is a family of periodic solutions bifurcates from the equilibrium point. Recently, Hajihosseini et al. [4] have investigated the following three-node network model:

$$
\begin{aligned}
\frac{d x_{1}(t)}{d t}= & -x_{1}(t)+\tanh \left[\int_{0}^{\infty} F(r) x_{2}(t-r) d r\right], \\
\frac{d x_{2}(t)}{d t}= & -x_{2}(t)+\tanh \left[\int_{0}^{\infty} F(r) x_{3}(t-r) d r\right], \\
\frac{d x_{3}(t)}{d t}= & -x_{3}(t)+w_{1} \tanh \left[\int_{0}^{\infty} F(r) x_{1}(t-r) d r\right] \\
& +w_{2} \tanh \left[\int_{0}^{\infty} F(r) x_{2}(t-r) d r\right],
\end{aligned}
$$

where $w_{1}$ and $w_{2}$ are parameters. The authors also make the change of variables $y_{i}(t)=\int_{0}^{\infty} F(r) x_{i}(t-r) d r(i=1,2,3)$ such that system (6) changes to the following:

$$
\begin{aligned}
\frac{d y_{1}(t)}{d t} & =-y_{1}(t)+\int_{0}^{\infty} F(r) \tanh \left[y_{2}(t-r)\right] d r, \\
\frac{d y_{2}(t)}{d t} & =-y_{2}(t)+\int_{0}^{\infty} F(r) \tanh \left[y_{3}(t-r)\right] d r, \\
\frac{d y_{3}(t)}{d t}= & -y_{3}(t)+w_{1} \int_{0}^{\infty} F(r) \tanh \left[y_{1}(t-r)\right] d r \\
& +w_{2} \int_{0}^{\infty} F(r) \tanh \left[y_{2}(t-r)\right] d r .
\end{aligned}
$$

Under the restrictive condition $\left|w_{1}+w_{2}\right|<1$, the Hopf bifurcation and stability of the bifurcating periodic solutions have been discussed. There are many authors who have studied the bifurcating periodic solutions for various models [5-14]. It is well known that the basic method in studying the bifurcating periodic solution for a time delay system is to discuss an algebraic equation in which the bifurcating value will be determined. Motivated by the above models we shall concern the existence of oscillating solutions for the following three-node network system with discrete and distributed delays:

$$
\begin{aligned}
\frac{d x_{1}(t)}{d t}= & -a_{1} x_{1}(t)+b_{2} f\left[x_{2}\left(t-\tau_{2}\right)\right] \\
& +c_{3} \int_{0}^{\infty} F(r) g\left[x_{3}(t-r)\right] d r \\
\frac{d x_{2}(t)}{d t}= & -a_{2} x_{2}(t)+b_{3} f\left[x_{3}\left(t-\tau_{3}\right)\right] \\
& +c_{1} \int_{0}^{\infty} F(r) g\left[x_{1}(t-r)\right] d r \\
\frac{d x_{3}(t)}{d t}= & -a_{3} x_{3}(t)+b_{1} f\left[x_{1}\left(t-\tau_{1}\right)\right] \\
& +c_{2} \int_{0}^{\infty} F(r) g\left[x_{2}(t-r)\right] d r,
\end{aligned}
$$

where $F(r)=\mu^{2} r e^{-\mu r}(\mu>0)$ is a strong kernel function, the passive decay rates $a_{1}, a_{2}$, and $a_{3}$ are positive constants, $b_{i}$, $c_{i}$, and $l_{i}(i=1,2,3)$ are constants, and delays $\tau_{1}, \tau_{2}$, and $\tau_{3}$ are nonnegative constants. It was emphasized that bifurcating approach is hard to deal with model (8), because it is very difficult to find the bifurcating parameter when $\tau_{1}, \tau_{2}$, and $\tau_{3}$ are different nonnegative constants. In order to discuss the existence of oscillating solutions for system (8) we adopt Chafee's criterion [15]. A time delay system will generate a limit cycle if the system has a unique unstable equilibrium point and bounded solutions. In other words, there exists an oscillatory solution of the model. System (8) can accord with the demands of Chafee's criterion; we refer the reader to [16, appendix]. 


\section{Preliminaries}

First we assume that the activation functions $f$ and $g$ both are monotone continuous bounded functions. $f, g \in C^{4}(R)$, $f(0)=g(0)=0, u f(u)>0$, and $u g(u)>0$ for $u \neq 0$. For example, $f(u)=\tanh (u)$, and $g(u)=\arctan (u)$ satisfy those conditions. For the kernel function $F(r)=\mu^{2} r e^{-\mu r}(\mu>0)$ we have

$$
\begin{aligned}
& \int_{0}^{\infty} F(r) g\left[x_{i}(t-r)\right] d r \\
&= \int_{0}^{\infty} \mu^{2} r e^{-\mu r} g\left[x_{i}(t-r)\right] d r \\
&= \int_{-\infty}^{t} \mu^{2}(t-s) e^{-\mu(t-s)} g\left[x_{i}(s)\right] d s \\
&= \mu^{2} e^{-\mu t}\left(t \int_{-\infty}^{t} e^{\mu s} g\left[x_{i}(s)\right] d s\right. \\
&\left.\quad-\int_{-\infty}^{t} s e^{\mu s} g\left[x_{i}(s)\right] d s\right) \quad(i=1,2,3) .
\end{aligned}
$$

From (9) we get

$$
\begin{aligned}
\frac{d}{d t} & \left\{\int_{0}^{\infty} F(r) g\left[x_{i}(t-r)\right] d r\right\} \\
= & -\mu \int_{0}^{\infty} F(r) g\left[x_{i}(t-r)\right] d r \\
& +\mu^{2} e^{-\mu t} \int_{-\infty}^{t} e^{\mu s} g\left[x_{i}(s)\right] d s \quad(i=1,2,3) .
\end{aligned}
$$

Taking the derivative on both sides of system (8) we obtain

$$
\begin{aligned}
\frac{d x_{1}^{2}(t)}{d t^{2}}= & -a_{1} \frac{d x_{1}(t)}{d t}+b_{2} f^{\prime}\left[x_{2}\left(t-\tau_{2}\right)\right] \frac{d x_{2}\left(t-\tau_{2}\right)}{d t} \\
& -\mu c_{3} \int_{0}^{\infty} F(r) g\left[x_{3}(t-r)\right] d r \\
& +c_{3} \mu^{2} e^{-\mu t} \int_{-\infty}^{t} e^{\mu s} g\left[x_{3}(s)\right] d s, \\
\frac{d x_{2}^{2}(t)}{d t^{2}}= & -a_{2} \frac{d x_{2}(t)}{d t}+b_{3} f^{\prime}\left[x_{3}\left(t-\tau_{3}\right)\right] \frac{d x_{3}\left(t-\tau_{3}\right)}{d t} \\
& -\mu c_{1} \int_{0}^{\infty} F(r) g\left[x_{1}(t-r)\right] d r \\
& +c_{1} \mu^{2} e^{-\mu t} \int_{-\infty}^{t} e^{\mu s} g\left[x_{1}(s)\right] d s, \\
\frac{d x_{3}^{2}(t)}{d t^{2}}= & -a_{3} \frac{d x_{3}(t)}{d t}+b_{1} f^{\prime}\left[x_{1}\left(t-\tau_{1}\right)\right] \frac{d x_{1}\left(t-\tau_{1}\right)}{d t} \\
& -\mu c_{2} \int_{0}^{\infty} F(r) g\left[x_{2}(t-r)\right] d r \\
& +c_{2} \mu^{2} e^{-\mu t} \int_{-\infty}^{t} e^{\mu s} g\left[x_{2}(s)\right] d s . \\
&
\end{aligned}
$$

Set

$$
\begin{aligned}
-\mu c_{3} & \int_{0}^{\infty} F(r) g\left[x_{3}(t-r)\right] d r \\
= & -2 \mu c_{3} \int_{0}^{\infty} F(r) g\left[x_{3}(t-r)\right] d r \\
& +\mu c_{3} \int_{0}^{\infty} F(r) g\left[x_{3}(t-r)\right] d r,
\end{aligned}
$$

so (11) can be rewritten as follows:

$$
\begin{aligned}
& \frac{d x_{1}^{2}(t)}{d t^{2}}=-a_{1} \frac{d x_{1}(t)}{d t}+b_{2} f^{\prime}\left[x_{2}\left(t-\tau_{2}\right)\right] \frac{d x_{2}\left(t-\tau_{2}\right)}{d t} \\
& -2 \mu\left[\frac{d x_{1}(t)}{d t}+a_{1} x_{1}(t)-b_{2} f\left[x_{2}\left(t-\tau_{2}\right)\right]\right] \\
& +\mu c_{3} \int_{0}^{\infty} F(r) g\left[x_{3}(t-r)\right] d r \\
& +c_{3} \mu^{2} e^{-\mu t} \int_{-\infty}^{t} e^{\mu s} g\left[x_{3}(s)\right] d s, \\
& \frac{d x_{2}^{2}(t)}{d t^{2}}=-a_{2} \frac{d x_{2}(t)}{d t}+b_{3} f^{\prime}\left[x_{3}\left(t-\tau_{3}\right)\right] \frac{d x_{3}\left(t-\tau_{3}\right)}{d t} \\
& -2 \mu\left[\frac{d x_{2}(t)}{d t}+a_{2} x_{2}(t)-b_{3} f\left[x_{3}\left(t-\tau_{3}\right)\right]\right] \\
& +\mu c_{1} \int_{0}^{\infty} F(r)\left[g x_{1}(t-r)\right] d r \\
& +c_{1} \mu^{2} e^{-\mu t} \int_{-\infty}^{t} e^{\mu s} g\left[x_{1}(s)\right] d s, \\
& \frac{d x_{3}^{2}(t)}{d t^{2}}=-a_{3} \frac{d x_{3}(t)}{d t}+b_{1} f^{\prime}\left[x_{1}\left(t-\tau_{1}\right)\right] \frac{d x_{1}\left(t-\tau_{1}\right)}{d t} \\
& -2 \mu\left[\frac{d x_{3}(t)}{d t}+a_{3} x_{3}(t)-b_{1} f\left[x_{1}\left(t-\tau_{1}\right)\right]\right] \\
& +\mu c_{2} \int_{0}^{\infty} F(r) g\left[x_{2}(t-r)\right] d r \\
& +c_{2} \mu^{2} e^{-\mu t} \int_{-\infty}^{t} e^{\mu s} g\left[x_{2}(s)\right] d s .
\end{aligned}
$$

Taking the derivative again on both sides of (13) and using (10) give

$$
\begin{aligned}
& \frac{d x_{1}^{3}(t)}{d t^{3}} \\
& =-a_{1} \frac{d x_{1}^{2}(t)}{d t^{2}}+b_{2} f^{\prime \prime}\left[x_{2}\left(t-\tau_{2}\right)\right]\left(\frac{d x_{2}\left(t-\tau_{2}\right)}{d t}\right)^{2} \\
& \quad+b_{2} f^{\prime}\left[x_{2}\left(t-\tau_{2}\right)\right] \frac{d x_{2}^{2}\left(t-\tau_{2}\right)}{d t^{2}}-2 \mu \frac{d x_{1}^{2}(t)}{d t^{2}} \\
& \quad-2 \mu a_{1} \frac{d x_{1}(t)}{d t}-2 \mu b_{2} f^{\prime}\left[x_{2}\left(t-\tau_{2}\right)\right] \frac{d x_{2}\left(t-\tau_{2}\right)}{d t}
\end{aligned}
$$




$$
\begin{aligned}
& -\mu^{2}\left(\frac{d x_{1}(t)}{d t}+a_{1} x_{1}(t)-b_{2} f\left[x_{2}\left(t-\tau_{2}\right)\right]\right) \\
& +c_{3} \mu^{2} g\left[x_{3}(t)\right] \\
& \frac{d x_{2}^{3}(t)}{d t^{3}} \\
& =-a_{2} \frac{d x_{2}^{2}(t)}{d t^{2}}+b_{3} f^{\prime \prime}\left[x_{3}\left(t-\tau_{3}\right)\right]\left(\frac{d x_{3}\left(t-\tau_{3}\right)}{d t}\right)^{2} \\
& +b_{3} f^{\prime}\left[x_{3}\left(t-\tau_{3}\right)\right] \frac{d x_{3}^{2}\left(t-\tau_{3}\right)}{d t^{2}}-2 \mu \frac{d x_{2}^{2}(t)}{d t^{2}} \\
& -2 \mu a_{2} \frac{d x_{2}(t)}{d t}-2 \mu b_{3} f^{\prime}\left[x_{3}\left(t-\tau_{3}\right)\right] \frac{d x_{3}\left(t-\tau_{3}\right)}{d t} \\
& -\mu^{2}\left(\frac{d x_{2}(t)}{d t}+a_{2} x_{2}(t)-b_{3} f\left[x_{3}\left(t-\tau_{3}\right)\right]\right) \\
& +c_{1} \mu^{2} g\left[x_{1}(t)\right], \\
& \frac{d x_{3}^{3}(t)}{d t^{3}} \\
& =-a_{3} \frac{d x_{3}^{2}(t)}{d t^{2}}+b_{1} f^{\prime \prime}\left[x_{1}\left(t-\tau_{1}\right)\right]\left(\frac{d x_{1}\left(t-\tau_{1}\right)}{d t}\right)^{2} \\
& +b_{1} f^{\prime}\left[x_{1}\left(t-\tau_{1}\right)\right] \frac{d x_{1}^{2}\left(t-\tau_{1}\right)}{d t^{2}}-2 \mu \frac{d x_{3}^{2}(t)}{d t^{2}} \\
& -2 \mu a_{3} \frac{d x_{3}(t)}{d t}-2 \mu b_{1} f^{\prime}\left[x_{1}\left(t-\tau_{1}\right)\right] \frac{d x_{1}\left(t-\tau_{1}\right)}{d t} \\
& -\mu^{2}\left(\frac{d x_{3}(t)}{d t}+a_{3} x_{3}(t)-b_{1} f\left[x_{1}\left(t-\tau_{1}\right)\right]\right) \\
& +c \mu^{2} g\left[x_{2}(t)\right] \text {. }
\end{aligned}
$$

Now by setting $x_{4}(t)=d x_{1}(t) / d t, x_{5}(t)=d x_{2}(t) / d t$, $x_{6}(t)=d x_{3}(t) / d t, x_{7}(t)=d x_{1}^{2}(t) / d t^{2}, x_{8}(t)=d x_{2}^{2}(t) / d t^{2}$, and $x_{9}(t)=d x_{3}^{2}(t) / d t^{2}$, we get the following time delay equivalent system of $(8)$ :

$$
\begin{aligned}
& \frac{d x_{1}(t)}{d t}=x_{4}(t), \\
& \frac{d x_{2}(t)}{d t}=x_{5}(t), \\
& \frac{d x_{3}(t)}{d t}=x_{6}(t), \\
& \frac{d x_{4}(t)}{d t}=x_{7}(t), \\
& \frac{d x_{5}(t)}{d t}=x_{8}(t),
\end{aligned}
$$

$$
\frac{d x_{6}(t)}{d t}=x_{9}(t)
$$$$
\frac{d x_{7}(t)}{d t}=-\mu^{2} a_{1} x_{1}(t)-\left(2 \mu a_{1}+\mu^{2}\right) x_{4}(t)
$$

$$
-\left(a_{1}+2 \mu\right) x_{7}(t)+b_{2} f^{\prime \prime}\left[x_{2}\left(t-\tau_{2}\right)\right]
$$$$
\times\left[x_{5}\left(t-\tau_{2}\right)\right]^{2}+b_{2} f^{\prime}\left[x_{2}\left(t-\tau_{2}\right)\right] x_{8}\left(t-\tau_{2}\right)
$$$$
-2 \mu b_{2} f^{\prime}\left[x_{2}\left(t-\tau_{2}\right)\right] x_{5}\left(t-\tau_{2}\right)
$$$$
+\mu^{2} b_{2} f\left[x_{2}\left(t-\tau_{2}\right)\right]+c_{3} \mu^{2} g\left[x_{3}(t)\right] \text {, }
$$$$
\frac{d x_{8}(t)}{d t}=-\mu^{2} a_{2} x_{2}(t)-\left(2 \mu a_{2}+\mu^{2}\right) x_{5}(t)
$$$$
-\left(a_{2}+2 \mu\right) x_{8}(t)+b_{3} f^{\prime \prime}\left[x_{3}\left(t-\tau_{3}\right)\right]
$$$$
\times\left[x_{6}\left(t-\tau_{3}\right)\right]^{2}+b_{3} f^{\prime}\left[x_{3}\left(t-\tau_{3}\right)\right] x_{9}\left(t-\tau_{3}\right)
$$$$
-2 \mu b_{3} f^{\prime}\left[x_{3}\left(t-\tau_{3}\right)\right] x_{6}\left(t-\tau_{3}\right)
$$$$
+\mu^{2} b_{3} f\left[x_{3}\left(t-\tau_{3}\right)\right]+c_{1} \mu^{2} g\left[x_{1}(t)\right] \text {, }
$$

$$
\begin{aligned}
\frac{d x_{9}(t)}{d t}= & -\mu^{2} a_{3} x_{3}(t)-\left(2 \mu a_{3}+\mu^{2}\right) x_{6}(t) \\
& -\left(a_{3}+2 \mu\right) x_{9}(t)+b_{1} f^{\prime \prime}\left[x_{1}\left(t-\tau_{1}\right)\right] \\
& \times\left[x_{4}\left(t-\tau_{1}\right)\right]^{2}+b_{1} f^{\prime}\left[x_{1}\left(t-\tau_{1}\right)\right] x_{7}\left(t-\tau_{1}\right) \\
& -2 \mu b_{1} f^{\prime}\left[x_{1}\left(t-\tau_{1}\right)\right] x_{4}\left(t-\tau_{1}\right) \\
& +\mu^{2} b_{1} f\left[x_{1}\left(t-\tau_{1}\right)\right]+c_{2} \mu^{2} g\left[x_{2}(t)\right] .
\end{aligned}
$$

The linearization of system (15) around the zero point is the following:

$$
\begin{aligned}
& \frac{d x_{1}(t)}{d t}=x_{4}(t), \\
& \frac{d x_{2}(t)}{d t}=x_{5}(t), \\
& \frac{d x_{3}(t)}{d t}=x_{6}(t), \\
& \frac{d x_{4}(t)}{d t}=x_{7}(t), \\
& \frac{d x_{5}(t)}{d t}=x_{8}(t), \\
& \frac{d x_{6}(t)}{d t}=x_{9}(t),
\end{aligned}
$$

$\frac{d x_{7}(t)}{d t}$

$$
=-\mu^{2} a_{1} x_{1}(t)-\left(2 \mu a_{1}+\mu^{2}\right) x_{4}(t)-\left(a_{1}+2 \mu\right) x_{7}(t)
$$




$$
\begin{gathered}
\quad+b_{2} f^{\prime}(0) x_{8}\left(t-\tau_{2}\right)-2 \mu b_{2} f^{\prime}(0) x_{5}\left(t-\tau_{2}\right) \\
+\mu^{2} b_{2} f^{\prime}(0) x_{2}\left(t-\tau_{2}\right)+c_{3} \mu^{2} g^{\prime}(0) x_{3}(t), \\
\frac{d x_{8}(t)}{d t} \\
=-\mu^{2} a_{2} x_{2}(t)-\left(2 \mu a_{2}+\mu^{2}\right) x_{5}(t)-\left(a_{2}+2 \mu\right) x_{8}(t) \\
+b_{3} f^{\prime}(0) x_{9}\left(t-\tau_{3}\right)-2 \mu b_{3} f^{\prime}(0) x_{6}\left(t-\tau_{3}\right) \\
\quad+\mu^{2} b_{3} f^{\prime}(0) x_{3}\left(t-\tau_{3}\right)+c_{1} \mu^{2} g^{\prime}(0) x_{1}(t), \\
\frac{d x_{9}(t)}{d t} \\
=-\mu^{2} a_{3} x_{3}(t)-\left(2 \mu a_{3}+\mu^{2}\right) x_{6}(t)-\left(a_{3}+2 \mu\right) x_{9}(t) \\
+b_{1} f^{\prime}(0) x_{7}\left(t-\tau_{1}\right)-2 \mu b_{1} f^{\prime}(0) x_{4}\left(t-\tau_{1}\right) \\
+\mu^{2} b_{1} f^{\prime}(0) x_{1}\left(t-\tau_{1}\right)+c_{2} \mu^{2} g^{\prime}(0) x_{2}(t) .
\end{gathered}
$$

System (16) can be written as a matrix form:

$$
\frac{d X(t)}{d t}=P X(t)+Q X(t-\widetilde{\tau}),
$$

where $X(t)=\left(x_{1}(t), x_{2}(t), \ldots, x_{9}(t)\right)^{T}, X(t-\widetilde{\tau})=\left(x_{1}\left(t-\tau_{1}\right)\right.$, $x_{2}\left(t-\tau_{2}\right), x_{3}\left(t-\tau_{3}\right), x_{4}\left(t-\tau_{1}\right), x_{5}\left(t-\tau_{2}\right), x_{6}\left(t-\tau_{3}\right), x_{7}\left(t-\tau_{1}\right)$, $x_{8}\left(t-\tau_{2}\right)$, and $\left.x_{9}\left(t-\tau_{3}\right)\right)^{T}$. So,

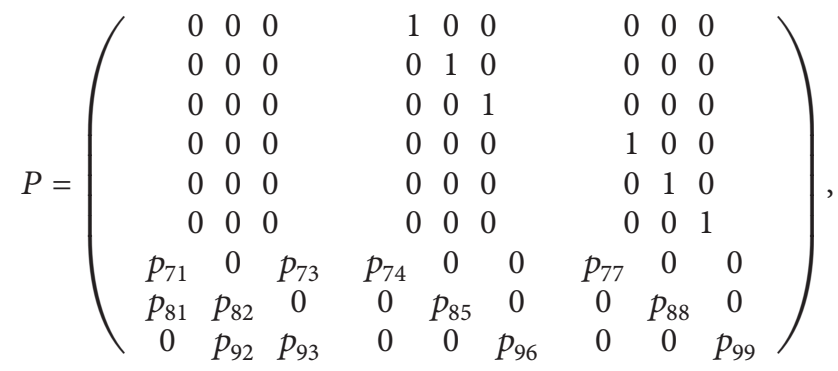

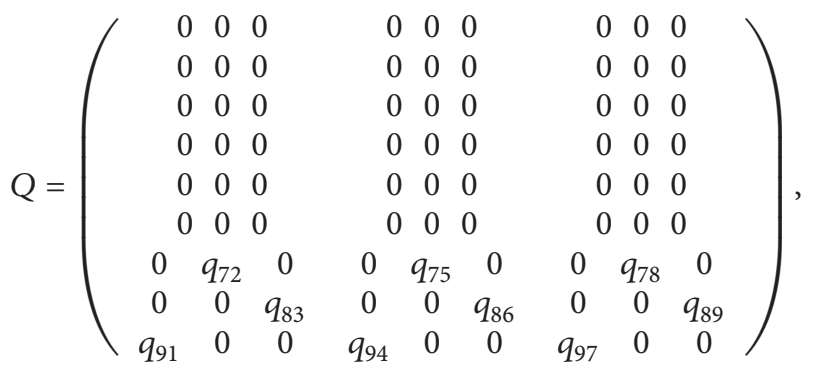

where $p_{71}=-\mu^{2} a_{1}, \quad p_{73}=c_{3} \mu^{2} g^{\prime}(0), p_{74}=-\left(2 \mu a_{1}+\right.$ $\left.\mu^{2}\right), p_{77}=-\left(a_{1}+2 \mu\right), \quad p_{81}=c_{1} \mu^{2} g^{\prime}(0), p_{82}=-\mu^{2} a_{2}$, $p_{85}=-\left(2 \mu a_{2}+\mu^{2}\right), \quad p_{88}=-\left(a_{2}+2 \mu\right), p_{92}=c_{2} \mu^{2} g^{\prime}(0)$, $p_{93}=-\mu^{2} a_{3}, p_{96}=-\left(2 \mu a_{3}+\mu^{2}\right), p_{99}=-\left(a_{3}+2 \mu\right)$; $q_{72}=\mu^{2} b_{2} f^{\prime}(0), q_{75}=-2 \mu b_{2} f^{\prime}(0), q_{78}=b_{2} f^{\prime}(0), q_{83}=$ $\mu^{2} b_{3} f^{\prime}(0), q_{86}=-2 \mu b_{3} f^{\prime}(0), q_{89}=b_{3} f^{\prime}(0), q_{91}=\mu^{2} b_{1} f^{\prime}(0)$, $q_{94}=-2 \mu b_{1} f^{\prime}(0)$, and $q_{97}=b_{1} f^{\prime}(0)$.
Lemma 1. All solutions of system (8) are bounded.

Proof. Since we assume that $f$ and $g$ both are monotone continuous bounded functions. So we have $|f(u)| \leq M$ and $|g(u)| \leq N$ ( $M$ and $N$ are positive constants). From (8) we obtain

$$
\begin{aligned}
& \frac{d\left|x_{1}(t)\right|}{d t} \leq-a_{1}\left|x_{1}(t)\right|+A_{1}, \\
& \frac{d\left|x_{2}(t)\right|}{d t} \leq-a_{2}\left|x_{2}(t)\right|+A_{2}, \\
& \frac{d\left|x_{3}(t)\right|}{d t} \leq-a_{3}\left|x_{3}(t)\right|+A_{3},
\end{aligned}
$$

where $A_{1}=\left|b_{2}\right| M+\left|c_{3}\right| N, A_{2}=\left|b_{3}\right| M+\left|c_{1}\right| N$, and $A_{3}=\left|b_{1}\right|$ $M+\left|c_{2}\right| N$. Thus, $\left|x_{i}(t)\right| \leq\left(A_{i} / a_{i}\right)+\left|x_{i}(0)\right|(i=1,2,3)$. This means that the solutions of system (8) are uniformly bounded.

Lemma 2. Assume that the matrix

$$
B=\left(\begin{array}{ccc}
-a_{1} & b_{2} f^{\prime}(0) & c_{3} g^{\prime}(0) \\
c_{1} g^{\prime}(0) & -a_{2} & b_{3} f^{\prime}(0) \\
b_{1} f^{\prime}(0) & c_{2} g^{\prime}(0) & -a_{3}
\end{array}\right)
$$

is a nonsingular matrix. Then system (8) has a unique equilibrium point.

Proof. Noting that $f$ and $g$ both are monotone continuous bounded functions satisfying $f(0)=g(0)=0$. Then $f(x)=$ $f^{\prime}(0) x+\alpha, g(x)=g^{\prime}(0) x+\beta$, where $\alpha$ and $\beta$ both are higher order infinitesimals when $x \rightarrow 0$. From (8), an equilibrium point $x^{*}=\left(x_{1}^{*}, x_{2}^{*}, x_{3}^{*}\right)^{T}$ is a solution of the following algebraic equation:

$$
\begin{aligned}
& -a_{1} x_{1}^{*}+b_{2} f\left(x_{2}^{*}\right)+c_{3} \int_{0}^{\infty} F(r) g\left(x_{3}^{*}\right) d r=0, \\
& -a_{2} x_{2}^{*}+b_{3} f\left(x_{3}^{*}\right)+c_{1} \int_{0}^{\infty} F(r) g\left(x_{1}^{*}\right) d r=0, \\
& -a_{3} x_{3}^{*}+b_{1} f\left(x_{1}^{*}\right)+c_{2} \int_{0}^{\infty} F(r) g\left(x_{2}^{*}\right) d r=0 .
\end{aligned}
$$

Noting that $\int_{0}^{\infty} F(r) g\left(x_{i}^{*}\right) d r=g\left(x_{i}^{*}\right) \int_{0}^{\infty} F(r) d r=$ $g\left(x_{i}^{*}\right)$, then we have

$$
\begin{aligned}
& -a_{1} x_{1}^{*}+b_{2} f^{\prime}(0) x_{2}^{*}+b_{2} \alpha+c_{3} g^{\prime}(0) x_{3}^{*}+c_{3} \beta=0, \\
& -a_{2} x_{2}^{*}+b_{3} f^{\prime}(0) x_{3}^{*}+b_{3} \alpha+c_{1} g^{\prime}(0) x_{1}^{*}+c_{1} \beta=0, \\
& -a_{3} x_{3}^{*}+b_{1} f^{\prime}(0) x_{1}^{*}+b_{1} \alpha+c_{2} g^{\prime}(0) x_{2}^{*}+c_{2} \beta=0 .
\end{aligned}
$$


If $y^{*}=\left(y_{1}^{*}, y_{2}^{*}, y_{3}^{*}\right)^{T}$ is another equilibrium point of system (8), neglecting of the higher order infinitesimal, we obtain

$$
\begin{aligned}
& -a_{1}\left(x_{1}^{*}-y_{1}^{*}\right)+b_{2} f^{\prime}(0)\left(x_{2}^{*}-y_{2}^{*}\right)+c_{3} g^{\prime}(0)\left(x_{3}^{*}-y_{3}^{*}\right)=0, \\
& -a_{2}\left(x_{2}^{*}-y_{2}^{*}\right)+b_{3} f^{\prime}(0)\left(x_{3}^{*}-y_{3}^{*}\right)+c_{1} g^{\prime}(0)\left(x_{1}^{*}-y_{1}^{*}\right)=0, \\
& -a_{3}\left(x_{3}^{*}-y_{3}^{*}\right)+b_{1} f^{\prime}(0)\left(x_{1}^{*}-y_{1}^{*}\right)+c_{2} g^{\prime}(0)\left(x_{2}^{*}-y_{2}^{*}\right) 0 .
\end{aligned}
$$

The matrix form of (23) is follows:

$$
B\left(x^{*}-y^{*}\right)=0 .
$$

Since $B$ is a nonsingular matrix based on the algebraic knowledge one can have $x^{*}-y^{*}=0$ or $x^{*}=y^{*}$. This means that system (8) has a unique equilibrium point. Obviously, the unique equilibrium point exactly is zero point since $f(0)=$ $g(0)=0$.

\section{Oscillating Solutions Analysis}

We adopt the following norms of vectors and matrices: $\|x(t)\|=\sum_{i=1}^{n}|x(t)|,\|P\|=\max _{j} \sum_{i=1}^{9}\left|p_{i j}\right|,\|Q\|=$ $\max _{j} \sum_{i=1}^{n}\left|q_{i j}\right|$. The measure $\sigma(P)$ of the matrix $P$ is defined by $\sigma(P)=\lim _{\theta \rightarrow 0^{+}}((\|I+\theta P\|-1) / \theta)$, which for the chosen norms reduces to $\sigma(P)=\max _{1 \leq j \leq 9}\left(p_{j j}+\sum_{i=1 i \neq j}^{9},\left|p_{i j}\right|\right)$.

In order to discuss the instability of equilibrium point for system (8), we consider the equivalent system (15) of (8). Note that the linearized system of (15) is (16). Obviously, if the trivial solution of (16) is unstable, it implies that the trivial solution of system (15) is unstable and thus the instability of the trivial solution of system (8). Therefore, we first have

Theorem 3. Assume that system (8) has a unique equilibrium point and the determinant of matrix $P$ is not equal to zero. Let $\rho_{1}, \rho_{2}, \ldots, \rho_{9}, \varrho_{1}, \varrho_{2}, \ldots, \varrho_{9}$ be the eigenvalues of the matrixes $P$ and $Q$, respectively. If there is at least one $\operatorname{Re} \rho_{i}>0\left(\operatorname{Im} \rho_{i}\right.$ may be equal to zero) or there exists one positive real eigenvalue $\varrho_{j}>\left|\rho_{j}\right|$, for some $j \in\{1,2, \ldots, 9\}$, then the unique equilibrium point, namely, the trivial solution of system (16) is unstable, implying that the equivalent system (15) or (8) generates oscillating solutions.

Proof. Since $Q$ is a singular matrix, set $\varrho_{1}=\varrho_{2}=\cdots=\varrho_{6}=0$. For given value of $\mu$, let $\rho_{1}, \rho_{2}, \ldots, \rho_{9}$ be the eigenvalues of the matrix $P$. Thus, the characteristic equation of system (16) is as follows:

$$
\operatorname{det}\left(\lambda I_{i j}-p_{i j}-q_{i j} e^{-\lambda \tau_{i j}}\right)=0
$$

where

$$
I_{i j}=\left\{\begin{array}{ll}
1 & \text { if } i=j, \\
0 & \text { if } i \neq j,
\end{array} \quad \tau_{i j}= \begin{cases}\tau_{i} & \text { if } i=j, \\
0 & \text { if } i \neq j .\end{cases}\right.
$$

or

$$
\prod_{i=1}^{9}\left[\lambda-\rho_{i}-\varrho_{i} e^{-\lambda \tau_{i}}\right]=0
$$

Since there is at least one $\operatorname{Re} \rho_{i}>0$, without loss of generality, set $\operatorname{Re} \rho_{1}>0$. From $\varrho_{1}=0$ we have the equation $\lambda-\rho_{1}=0$. This means that $\lambda=\rho_{1}$, and there is a $\operatorname{Re} \lambda>0$. So, the unique equilibrium point of system (16) is unstable based on the theory of differential equation. If by corresponding some $\rho_{j}$ we have $\varrho_{j}>\left|\rho_{j}\right| j \in\{1,2, \ldots, 9\}$, we pointed out that there exists positive real root for transcendental equation $\lambda-\rho_{j}-\varrho_{j} e^{-\lambda \tau_{j}}=0$. Let $f(\lambda)=\lambda-\rho_{j}-\varrho_{j} e^{-\lambda \tau_{j}}$. Then $f(\lambda)$ is a continuous function of $\lambda$. Since $f(0)=-\rho_{j}-\varrho_{j}$, from $\varrho_{j}>\left|\rho_{j}\right|$, we know that $f(0)<0$. Obviously, there is a suitable large $\lambda^{*}>0$ such that $f\left(\lambda^{*}\right)=\lambda^{*}-\rho_{j}-\varrho_{j} e^{-\lambda^{*} \tau_{j}}>0$ since $e^{-\lambda^{*} \tau_{j}}$ can be suitably small. Thus, by the continuity of $f(\lambda)$, there is a point, say $\bar{\lambda} \in\left(0, \lambda^{*}\right)$ such that $f(\bar{\lambda})=0$. In other words, there is a positive real eigenvalue. In this case the unique equilibrium point of system (16) is also unstable. Since all solutions of the system are bounded, on the basis of Chafee's criterion [15], system (15) generates a limit cycle, implying that there exists an oscillating solution of system (15) and therefore system (8).

Theorem 4. Assume that system (8) has a unique equilibrium point and the following inequalities hold:

$$
\begin{gathered}
(\|Q\| \tau e) \exp (-|\sigma(P)| \tau)>1, \\
\left(\|Q\| \tau^{*} e\right) \exp \left(-\tau^{*}|\sigma(P)| \tau^{*}\right)>1,
\end{gathered}
$$

where $\tau=\min \left\{\tau_{1}, \tau_{2}, \tau_{3}\right\}$ and $\tau^{*}=\max \left\{\tau_{1}, \tau_{2}, \tau_{3}\right\}$. Then the unique equilibrium point, namely, the trivial solution of system (16) is unstable, implying that the equivalent system (15) or (8) generates oscillating solutions.

Proof. First we consider system (16) in the case $\tau_{1}=\tau_{2}=\tau_{3}=$ $\tau$ and we easily get:

$$
\begin{aligned}
& \frac{d\left|x_{1}(t)\right|}{d t}=\left|x_{4}(t)\right|, \\
& \frac{d\left|x_{2}(t)\right|}{d t}=\left|x_{5}(t)\right|, \\
& \frac{d\left|x_{3}(t)\right|}{d t}=\left|x_{6}(t)\right|, \\
& \frac{d\left|x_{4}(t)\right|}{d t}=\left|x_{7}(t)\right|, \\
& \frac{d\left|x_{5}(t)\right|}{d t}=\left|x_{8}(t)\right|, \\
& \frac{d\left|x_{6}(t)\right|}{d t}=\left|x_{9}(t)\right|,
\end{aligned}
$$

$$
\begin{aligned}
\frac{d\left|x_{7}(t)\right|}{d t} \leq & -\mu^{2} a_{1}\left|x_{1}(t)\right|-\left(2 \mu a_{1}+\mu^{2}\right)\left|x_{4}(t)\right| \\
& -\left(a_{1}+2 \mu\right)\left|x_{7}(t)\right|+\left|b_{2} f^{\prime}(0)\right|\left|x_{8}(t-\tau)\right| \\
& +\left|2 \mu b_{2} f^{\prime}(0)\right|\left|x_{5}\left(t-\tau_{2}\right)\right| \\
& +\left|\mu^{2} b_{2} f^{\prime}(0)\right|\left|x_{2}\left(t-\tau_{2}\right)\right|+\left|c_{3} \mu^{2} g^{\prime}(0)\right|\left|x_{3}(t)\right|,
\end{aligned}
$$




$$
\begin{aligned}
\frac{d\left|x_{8}(t)\right|}{d t} \leq-\mu^{2} a_{2}\left|x_{2}(t)\right|-\left(2 \mu a_{2}+\mu^{2}\right)\left|x_{5}(t)\right| \\
-\left(a_{2}+2 \mu\right)\left|x_{8}(t)\right|+\left|b_{3} f^{\prime}(0)\right|\left|x_{9}\left(t-\tau_{3}\right)\right| \\
+\left|2 \mu b_{3} f^{\prime}(0)\right|\left|x_{6}\left(t-\tau_{3}\right)\right| \\
+\left|\mu^{2} b_{3} f^{\prime}(0)\right|\left|x_{3}\left(t-\tau_{3}\right)\right|+\left|c_{1} \mu^{2} g^{\prime}(0)\right|\left|x_{1}(t)\right|, \\
\frac{d\left|x_{9}(t)\right|}{d t} \leq-\mu^{2} a_{3}\left|x_{3}(t)\right|-\left(2 \mu a_{3}+\mu^{2}\right)\left|x_{6}(t)\right| \\
\quad-\left(a_{3}+2 \mu\right)\left|x_{9}(t)\right|+\left|b_{1} f^{\prime}(0)\right|\left|x_{7}(t-\tau)\right| \\
+\left|2 \mu b_{1} f^{\prime}(0)\right|\left|x_{4}\left(t-\tau_{1}\right)\right| \\
+\left|\mu^{2} b_{1} f^{\prime}(0)\right|\left|x_{1}\left(t-\tau_{1}\right)\right|+\left|c_{2} \mu^{2} g^{\prime}(0)\right|\left|x_{2}(t)\right| .
\end{aligned}
$$

Let $(t)=\sum_{i=1}^{9}\left|x_{i}(t)\right| ;$ then for some $t^{*}, y(t)>0\left(t \geq t^{*}\right)$ and we have

$$
\frac{d y(t)}{d t} \leq \sigma(P) y(t)+\|Q\| y(t-\tau), \quad t \geq t^{*}+\tau .
$$

Consider the scalar equation

$$
\frac{d z(t)}{d t}=\sigma(P) z(t)+\|Q\| z(t-\tau), \quad t \geq t^{*}+\tau
$$

with $y(s)=z(s)$ and $s \in\left[t^{*}, t^{*}+\tau\right]$. According to the comparison theorem of differential equation, one can obtain

$$
y(t) \leq z(t), \quad t \geq t^{*}+\tau
$$

We claim that the trivial solution of (31) is unstable. Suppose that this is not true; then the characteristic equation associated with (31) given by

$$
\lambda=\sigma(P)+\|Q\| e^{-\lambda \tau}
$$

will have a real negative root say $\lambda^{*}$ such that $\lambda^{*}=\sigma(P)+$ $\|\mathrm{Q}\| e^{-\lambda^{*} \tau}$, where $e^{-\lambda^{*} \tau}=e^{\left|\lambda^{*} \tau\right|}$.

So we get

$$
\left|\lambda^{*}\right| \geq\|Q\| e^{\left|\lambda^{*} \tau\right|}-|\sigma(P)|
$$

Thus

$$
\begin{aligned}
1 & \geq \frac{\|Q\| e^{\left|\lambda^{*} \tau\right|}}{\left|\lambda^{*}\right|+|\sigma(P)|} \\
& =\frac{\|Q\| \tau \exp (-|\sigma(P)| \tau) \exp \left(\left|\lambda^{*}\right| \tau+|\sigma(P) \tau|\right)}{\left|\lambda^{*}\right| \tau+|\sigma(P) \tau|} .
\end{aligned}
$$

Based on the formula $e^{x} \geq e x(x>0)$, from (35), we have

$$
1 \geq(\|Q\| \tau e) \exp (-|\sigma(P)| \tau) .
$$

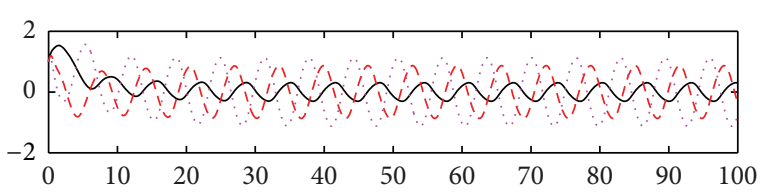

(a) solid line: $x_{1}(t)$, dashed line: $x_{2}(t)$, and dotted line: $x_{3}(t)$

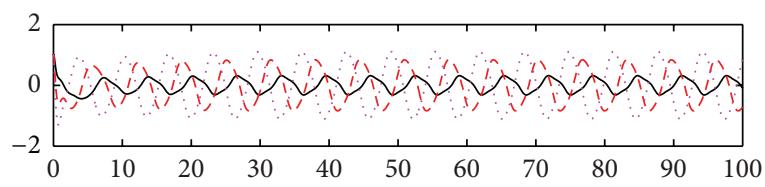

(b) solid line: $x_{4}(t)$, dashed line: $x_{5}(t)$, and dotted line: $x_{6}(t)$

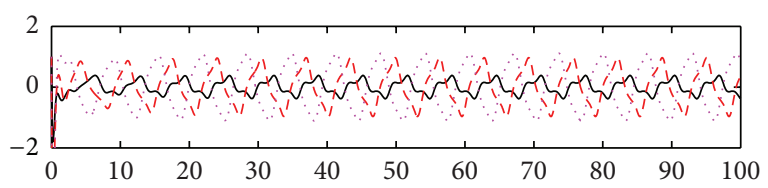

(c) solid line: $x_{7}(t)$, dashed line: $x_{8}(t)$, and dotted line: $x_{9}(t)$

FIGURE 1: Oscillatory behavior of the solutions: $m u=3.5$, delays: $(0.5,0.6$, and 0.7), and activation functions: $f(u)=g(u)=\tanh (u)$.

Inequality (36) contradicts the first inequality of (28). Therefore, our claim regarding the instability of the trivial solution is valid. Similarly, consider system (16) in the case $\tau_{1}=\tau_{2}=\tau_{3}=\tau^{*}$; we know that the trivial solution is unstable if the second inequality of (28) holds. Note that $\tau \leq \tau_{i} \leq \tau^{*}(i=1,2,3)$. So, the trivial solution of (16) is unstable when condition (28) holds, implying that the trivial solution of (15) is unstable. According to Chafee's criterion, system (15) generates a limit cycle, suggesting that there is an oscillating solution of system (15) and therefore system (8).

\section{Computer Simulations}

We use the equivalent system (15) of (8) for computer simulation. In Figure 1 both activation functions $f(u)$ and $g(u)$ are taken $\tanh (u)$. Thus, $f^{\prime}(u)=g^{\prime}(u)=4 /\left(e^{u}+e^{-u}\right)^{2}=$ $1-(\tanh (u))^{2}, f^{\prime \prime}(u)=g^{\prime \prime}(u)=2(\tanh (u))^{3}-2 \tanh (u)$, and $f^{\prime}(0)=g^{\prime}(0)=1$. We select $\mu=5.5, a_{1}=0.48, a_{2}=0.65$, $a_{3}=0.78, b_{1}=0.6, b_{2}=0.8, b_{3}=0.9, c_{1}=-0.8$, and $c_{2}=-1.8, c_{3}=0.4$. Then the eigenvalues of matrix $P$ are $-5.8250 \pm 1.8620 i,-1.7568 \pm 2.5151 i,-0.0000$, $-0.4840,-5.3568,-5.6392$, and -8.2087 and the eigenvalues of matrix $Q$ are $-0.3780 \pm 0.6547 i, 0.7560$, and $0,0,0,0,0,0$. Note that the eigenvalue 0.7560 of matrix $Q$ is larger than $|-0.4840|=0.4840$. Based on Theorem 3, the trivial solution of system (15) is unstable. The system generates an oscillating solution. In order to compare the effect of the time delays, we change the delays from $(0.2,0.3$, and 0.4$)$ to $(1,1.5$, and 2), while keeping the other parameters as shown in Figure 1. We see that the oscillatory amplitude and frequency both are changed (see Figure 2). In Figure 3, we keep the parameter values the same as Figure 2, the activation function $g(u)=\tanh (u)$, and change the activation function $f(u)=$ $\arctan (u)$. Then $f^{\prime}(u)=1 /\left(1+u^{2}\right)$ and $f^{\prime \prime}(u)=-2 u /(1+$ 


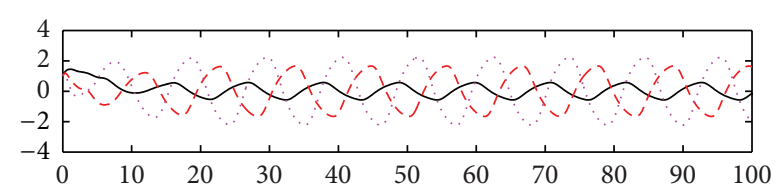

(a) solid line: $x_{1}(t)$, dashed line: $x_{2}(t)$, and dotted line: $x_{3}(t)$

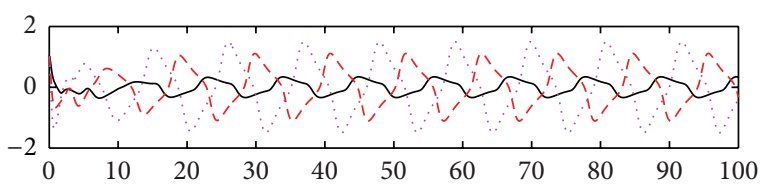

(b) solid line: $x_{4}(t)$, dashed line: $x_{5}(t)$, and dotted line: $x_{6}(t)$

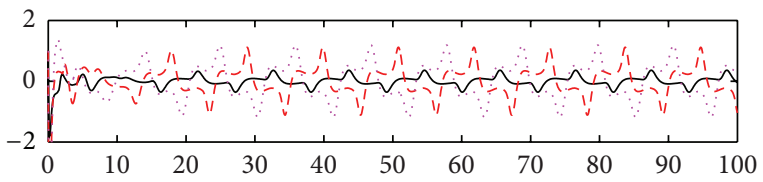

(c) solid line: $x_{7}(t)$, dashed line: $x_{8}(t)$, and dotted line: $x_{9}(t)$

FIGURE 2: Oscillatory behavior of the solutions: $m u=5.5$, delays: $(1$, 1.5 , and 2), and activation functions: $f(u)=g(u)=\tanh (u)$.

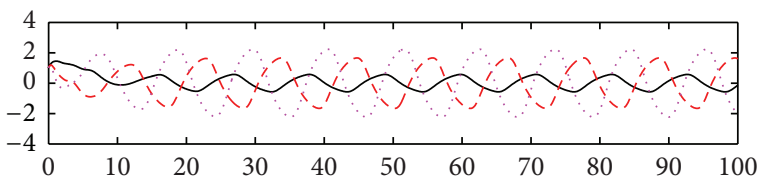

(a) solid line: $x_{1}(t)$, dashed line: $x_{2}(t)$, and dotted line: $x_{3}(t)$

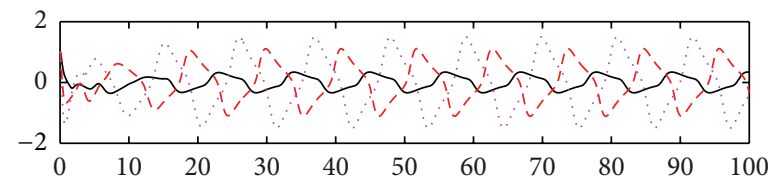

(b) solid line: $x_{4}(t)$, dashed line: $x_{5}(t)$, and dotted line: $x_{6}(t)$

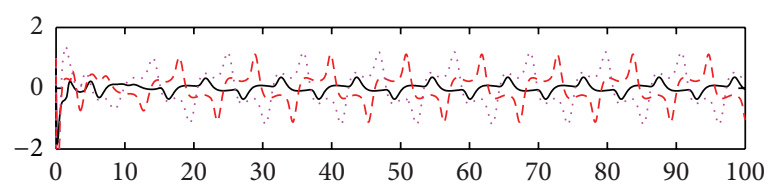

(c) solid line: $x_{7}(t)$, dashed line: $x_{8}(t)$, and dotted line: $x_{9}(t)$

FIGURE 3: Oscillatory behavior of the solutions: $m u=5.5$, delays: $(1,1.5$, and 2), and activation functions: $f(u)=\arctan (u), g(u)=$ $\tanh (u)$.

$\left.u^{2}\right)^{2}$. We see that the graph is changed slightly in comparison with Figure 2. In Figure 4, we set the activation function $f(u)=1 /\left(1+e^{-2 u}\right)$ and $g(u)=\left(e^{-2 u}-1\right) /\left(1+e^{-2 u}\right)$. Then $f^{\prime}(u)=2 e^{-2 u} /\left(1+e^{-2 u}\right)^{2}, g^{\prime}(u)=-4 e^{-2 u} /\left(1+e^{-2 u}\right)^{2}$, $f^{\prime \prime}(u)=4 e^{-2 u}\left(2 e^{-2 u}-1\right) /\left(1+e^{-2 u}\right)^{3}$, and $g^{\prime \prime}(u)=8 e^{-2 u}(1-$ $\left.e^{-2 u}\right) /\left(1+e^{-2 u}\right)^{3}$. Thus $f^{\prime}(0)=1 / 2$ and $g^{\prime}(0)=-1$. Note that $f(0)=1 / 2 \neq 0$ and $g(0)=-1 / 2 \neq 0$. When we select the parameters as follows: $\mu=3.5, a_{1}=0.18, a_{2}=0.15$, $a_{3}=0.19, b_{1}=0.05, b_{2}=0.08, b_{3}=0.12, c_{1}=-0.6$,

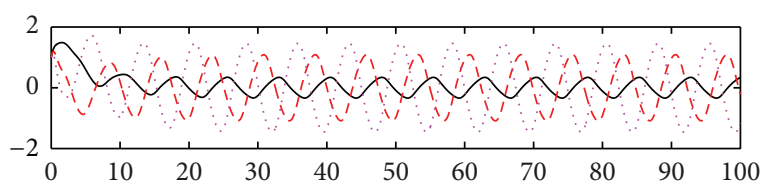

(a) solid line: $x_{1}(t)$, dashed line: $x_{2}(t)$, and dotted line: $x_{3}(t)$

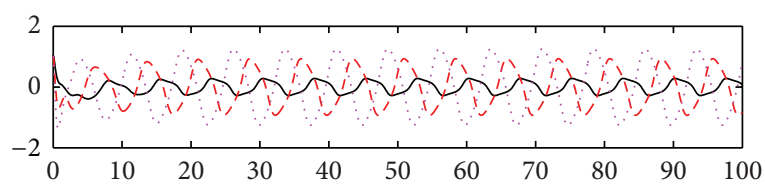

(b) solid line: $x_{4}(t)$, dashed line: $x_{5}(t)$, and dotted line: $x_{6}(t)$

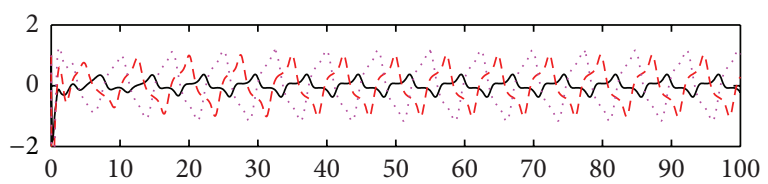

(c) solid line: $x_{7}(t)$, dashed line: $x_{8}(t)$, and dotted line: $x_{9}(t)$

FIGURE 4: Oscillatory behavior of the solutions: $m u=3.5$, delays: $(0.5,0.6$, and 0.7$)$, and activation functions: $f(u)=1 /(1+\exp (-2 u))$, $g(u)=(\exp (-2 u)-1) /(1+\exp (-2 u))$.

and $c_{2}=0.8, c_{3}=0.5$, time delays are $(0.5,0.6$, and 0.7$)$. The oscillatory solutions also appeared. This means that the restrictive condition $f(0)=g(0)=0, u f(u)>0$, and $u g(u)>$ 0 for $u \neq 0$ is only for convenience of the proof.

\section{Conclusion}

This paper discusses the oscillatory behavior of the solutions for a three-node network model with discrete and distributed delays. Two theorems are provided to ensure the existence of oscillating solutions for the model. Computer simulations suggested that our theorems are only sufficient conditions. How to find a necessary condition is still an open problem.

\section{Conflict of Interests}

The author declares that there is no conflict of interests regarding the publication of this paper.

\section{Acknowledgment}

This work is supported by Grant no. 11361010 from NNFS of China to Chunhua Feng.

\section{References}

[1] K. Gopalsamy and I. K. C. Leung, "Convergence under dynamical thresholds with delays," IEEE Transactions on Neural Networks, vol. 8, no. 2, pp. 341-348, 1997.

[2] S. Ruan and R. S. Filfil, "Dynamics of a two-neuron system with discrete and distributed delays," Physica D, vol. 191, no. 3-4, pp. 323-342, 2004. 
[3] X. Liao, S. Li, and G. Chen, "Bifurcation analysis on a twoneuron system with distributed delays in the frequency domain," Neural Networks, vol. 17, no. 4, pp. 545-561, 2004.

[4] A. Hajihosseini, G. R. Lamooki, B. Beheshti, and F. Maleki, "The Hopf bifurcation analysis on a time-delayed recurrent neural network in the frequency domain," Neurocomputing, vol. 73, pp. 991-1005, 2010.

[5] T. Y. Cai, H. G. Zhang, and F. H. Yang, "Simplified frequency meth-od for stability and bifurcation of delayed neural networks in ring structure," Neurocomputing, vol. 121, pp. 416-422, 2013.

[6] R. M. Sebdani and S. Farjami, "Bifurcations and chaos in a discrete-time-delayed Hopfield neural network with ring structures and different internal delays," Neurocomputing, vol. 99, pp. 154-162, 2013.

[7] C. Huang, L. Huang, J. Feng, M. Nai, and Y. He, "Hopf bifurcation analysis for a two-neuron network with four delays," Chaos, Solitons and Fractals, vol. 34, no. 3, pp. 795-812, 2007.

[8] Y. T. Ding, W. H. Jiang, and P. Yu, "Bifurcation analysis in a recurrent neural network model with delays," Communications in Nonlinear Science and Numerical Simulation, vol. 18, pp. 351372, 2013.

[9] S. Guo, Y. Chen, and J. Wu, "Two-parameter bifurcations in a network of two neurons with multiple delays," Journal of Differential Equations, vol. 244, no. 2, pp. 444-486, 2008.

[10] Y. Huang and X. Yang, "Hyperchaos and bifurcation in a new class of four-dimensional Hopfield neural networks," Neurocomputing, vol. 69, no. 13-15, pp. 1787-1795, 2006.

[11] C. Sun, M. Han, and X. Pang, "Global Hopf bifurcation analysis on a BAM neural network with delays," Physics Letters A, vol. 360, no. 6, pp. 689-695, 2007.

[12] H. Zhao, L. Wang, and C. Ma, "Hopf bifurcation and stability analysis on discrete-time Hopfield neural network with delay," Nonlinear Analysis: Real World Applications, vol. 9, no. 1, pp. 103-113, 2008.

[13] I. Ncube, "Stability switching and Hopf bifurcation in a multiple-delayed neural network with distributed delay," Journal of Mathematical Analysis and Applications, vol. 407, pp. 141-146, 2013.

[14] T. Dong and X. F. Liao, "Hopf-Pitchfork bifurcation in a simplified BAM neural network model with multiple delays," Journal of Computational and Applied Mathematics, vol. 253, pp. 222234, 2013.

[15] N. Chafee, "A bifurcation problem for a functional differential equation of finitely retarded type," Journal of Mathematical Analysis and Applications, vol. 35, no. 2, pp. 312-348, 1971.

[16] C. Feng and R. Plamondon, "An oscillatory criterion for a time delayed neural ring network model," Neural Networks, vol. 2930, pp. 70-79, 2012. 

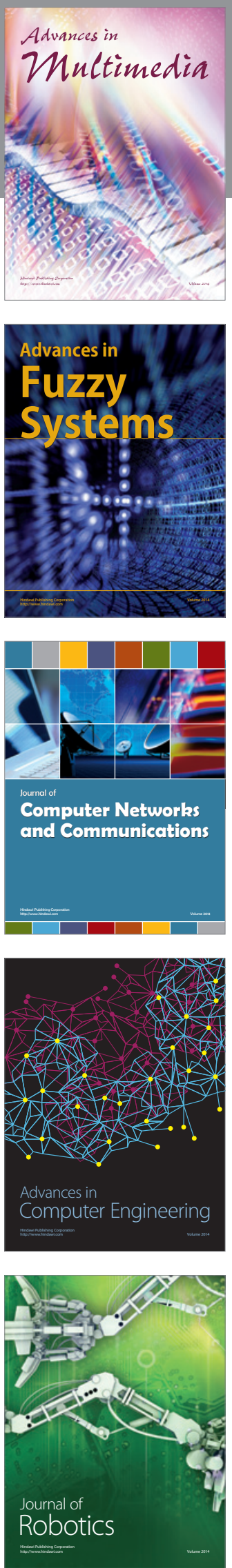

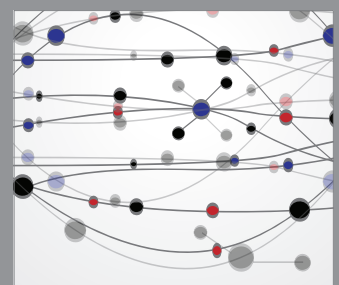

The Scientific World Journal
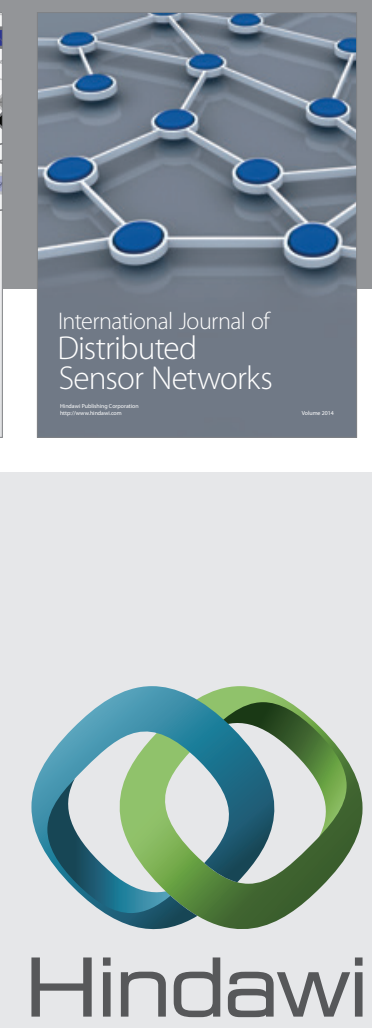

Submit your manuscripts at

http://www.hindawi.com
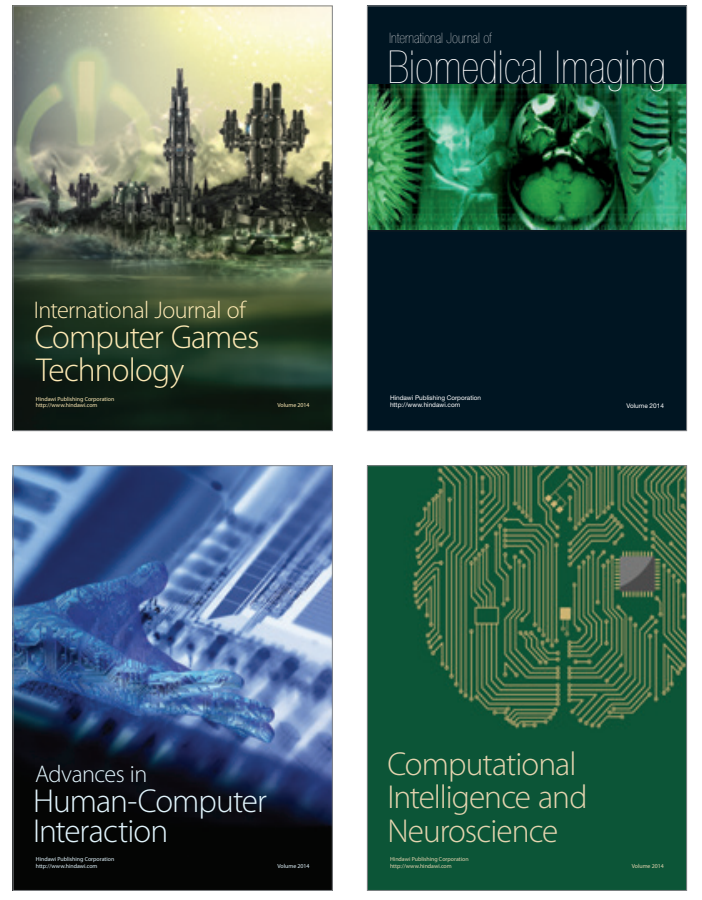
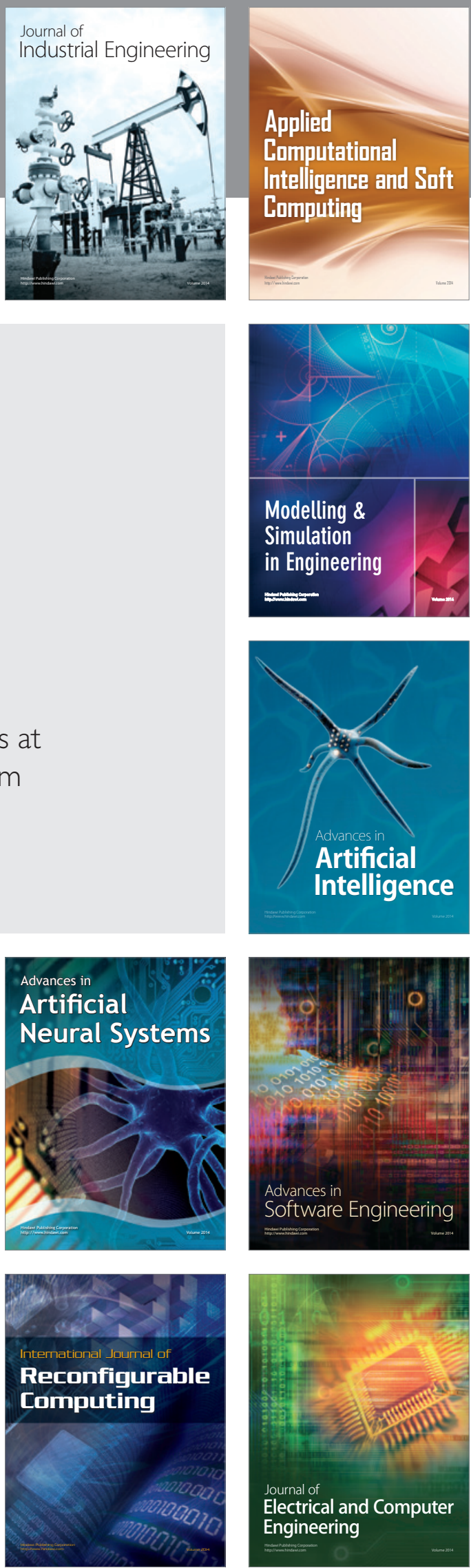